\title{
C-reactive protein and albumin association with mortality of hospitalised SARS-CoV-2 patients: A tertiary hospital experience
}

\author{
Authors: Ayman S Bannaga, ${ }^{\mathrm{A}}$ Maria Tabuso, ${ }^{\mathrm{B}}$ Alexia Farrugia, ${ }^{\mathrm{C}}$ Subashini Chandrapalan, ${ }^{\mathrm{D}}$ Karenjit Somal, ${ }^{\mathrm{E}}$ \\ Voon Kune Lim, ${ }^{\mathrm{F}}$ Shahd Mohamed, ${ }^{\mathrm{G}}$ Gohar $]$ Nia, ${ }^{\mathrm{G}}$ Jayan Mannath, ${ }^{\mathrm{H}}$ John LH Wong, ${ }^{\mathrm{I}}$ Angela Noufaily, ${ }^{\mathrm{J}}$ \\ Benjamin R Disney ${ }^{\mathrm{H}}$ and Ramesh P Arasaradnam ${ }^{\mathrm{K}}$
}

\section{Objective}

The objective was to study hospitalised COVID-19 patients' mortality and intensive care unit (ICU) admission with covariates of interest (age, gender, ethnicity, clinical presentation, comorbidities and admission laboratory findings).

Methods

Logistic regression analyses were performed for patients admitted to University Hospital, University Hospitals Coventry and Warwickshire NHS Trust, between 24 January 2020 - 13 April 2020

Results

There were 321 patients hospitalised. Median age was 73 years and $189(59 \%)$ were male. Ethnicity was divided between Caucasian (77\%), and black, Asian, and minority ethnic (BAME) groups (23\%). Commonest symptoms were dyspnoea (62.9\%), fever (59.1\%) and cough (56\%). Gastrointestinal symptoms amounted to $11.8 \%$.

Authors: ${ }^{\text {A }}$ clinical research fellow in gastroenterology and hepatology, University Hospitals Coventry and Warwickshire NHS Trust, Coventry, UK and Warwick Medical School, Coventry, UK; ${ }^{B}$ Clinical research fellow in gastroenterology, University Hospitals Coventry and Warwickshire NHS Trust, Coventry, UK; C Clinical research fellow in general surgery, University Hospitals Coventry and Warwickshire NHS Trust, Coventry, UK and Warwick Medical School, Coventry, UK; ' Clinical research fellow in gastroenterology, University Hospitals Coventry and Warwickshire NHS Trust, Coventry,

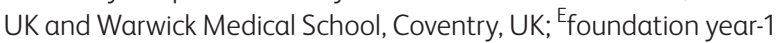
trainee, University Hospitals Coventry and Warwickshire NHS Trust, Coventry, UK; F core medical trainee, University Hospitals Coventry and Warwickshire NHS Trust, Coventry, UK; ${ }^{G}$ speciality trainee in gastroenterology and general medicine, University Hospitals Coventry and Warwickshire NHS Trust, Coventry, UK; ${ }^{{ }^{H}}$ consultant gastroenterologist, University Hospitals Coventry and Warwickshire NHS Trust, Coventry, UK; ' ${ }^{\mathrm{C}}$ consultant gastroenterologist and hepatologist, University Hospitals Coventry and Warwickshire NHS Trust, Coventry, UK; ' senior research fellow in medical statistics, Warwick Medical School, Coventry, UK; ${ }^{\text {K}}$ consultant gastroenterologist, University Hospitals Coventry and Warwickshire NHS Trust, Coventry, UK and Warwick Medical School, Coventry, UK
Forty-four patients (13.7\%) received ICU care. ICU male to female ratio was $3: 1$ ( $p=0.027$; odds ratio (OR) $2.3 ; 95 \%$ confidence interval (CI) 1.1-4.9), BAME ( $p=0.008$; OR 2.5; $95 \%$ CI $1.3-4.9)$, age $>65$ years $(p=0.026$; OR $0.28 ; 95 \%$ CI $0.09-0.93$ ), heart disease ( $p=0.009$; OR $0.2 ; 95 \%$ CI $0.1-0.6$ ) and elevated C-reactive protein (CRP; $p<0.001$; OR 1.004; 95\% CI 1.002-1.008) were associated with ICU admission.

One-hundred and four patients (32.4\%) died. Age $>65$ years $(p=0.011 ;$ OR 5 ; 95\% CI 1.6-21.9), neutrophils $(p=0.047)$, neutrophil:lymphocyte ratio (NLR; $p=0.028), \operatorname{CRP}(p<0.001)$ and albumin $(p=0.002)$ were associated with mortality. When analysis adjusted for age, CRP $(p<0.001$; OR 1.006; $95 \%$ CI 1.004-1.008) and albumin ( $p=0.005$; OR 0.94; $95 \%$ CI 0.90-0.98) remained associated with mortality.

\section{Conclusions}

COVID-19 has high mortality. BAME and male patients were associated with ICU admission. High CRP and low albumin (after correcting for age) were associated with mortality.

KEYWORDS: SARS-CoV-2, COVID-19, mortality, C-reactive protein, albumin

DOI: $10.7861 /$ clinmed.2020-0424

\section{Introduction}

Severe acute respiratory syndrome coronavirus-2 (SARS-CoV-2) was first described in Wuhan in December 2019 which rapidly spread to 215 countries, areas and territories leading to a pandemic. ${ }^{1}$ This has invariably resulted in implementing key preventive measures globally, including social distancing. The UK government implemented social distancing measures on 23 March 2020 . However, at the time of writing, daily deaths were still on the rise. ${ }^{2}$ In the UK, early reports show significant regional variation and the Midlands has reported both higher incidence and death rate next to London. ${ }^{2}$

The high basic reproductive number $\left(R_{0}\right)$ and its shorter serial interval render SARS-CoV-2 and the coronavirus disease (COVID-19) as a highly contagious infection. ${ }^{3,4}$ The most common transmission modality includes droplet inhalation and contact transmission. However, concerns have been raised with regards 
to the possibility of faecal-oral spread. Several studies have confirmed the presence of viral ribonucleic acid (RNA) in faecal samples, even after negative sputum. ${ }^{5}$

The most described symptoms of COVID-19 are fever, cough and shortness of breath. ${ }^{6}$ Further reports emerged showing that non-respiratory symptoms, such as loss of taste and/or smell, could also be a presenting clinical feature. ${ }^{7}$ Although initially under-reported, gastrointestinal (GI) symptoms have been increasingly recognised with the variable prevalence of 3-61\% across studies. ${ }^{8-11}$ Anorexia was the most commonly reported GI symptom, followed by diarrhoea and vomiting. ${ }^{12}$ In addition to COVID-19 symptomatology, other parameters that could stratify patients that require close monitoring include the admission biochemical abnormalities. ${ }^{13}$

Several mechanisms have been hypothesised for COVID-19 pathogenicity. Cellular entry of the viral RNA through the interaction with angiotensin converting enzyme-2 (ACE-2) receptors is the commonly described one. It also remains unclear how the virus particles result in GI symptoms. ${ }^{8-12}$

At present, there is a scarcity of data looking at the clinical characteristics of the COVID-19 patients in the UK. In this study, we performed a comprehensive analysis on clinical characteristics of the patients with COVID-19 with the aim of identifying the high-risk patient group early.

\section{Methods}

\section{Study design and participants}

This was a single centre, retrospective study at University Hospital, University Hospitals Coventry and Warwickshire NHS Trust. The data was retrieved from the electronic hospital informatics system. We looked into a cohort of hospitalised patients who presented to the hospital from 24 January 2020 leading up to the peak of UK epidemic by 13 April 2020. A confirmed case was defined as having a positive result for SARS-CoV-2 on real-time reverse-transcription - polymerase chain reaction (RT-PCR) assay of either nasal and pharyngeal swab or sputum specimens. Ethical approval was obtained from University Hospitals Coventry and Warwickshire NHS Trust Research and Development Department (Ref GF0926). The study conformed to the Declaration of Helsinki that was developed by the World Medical Association.

\section{Data management and analysis}

The data on demographic details of the patients, presenting symptoms, comorbidities and admission blood tests were obtained from the hospital electronic informatics system. The blood work-up at admission included full blood count, renal function tests, liver function tests and C-reactive protein (CRP). The data on radiological investigations were obtained from the official radiological reports by a consultant radiologist. In the absence of this, a documentation in the electronic case records by a physician was considered sufficient. All of the included patients were followed up during their hospital stay until they were discharged or died.

The association of hospital care (standard ward care or intensive care unit (ICU) admission) and mortality (dead or alive at hospital discharge) with covariates of interest (age, gender, ethnicity, haemoglobin, neutrophil count, lymphocyte count, neutrophil lymphocyte ratio, platelets count, CRP, alanine aminotransferase (ALT), albumin, bilirubin, creatinine, comorbidity, diabetes, hypertension, heart disease, inflammatory bowel disease, liver disease and history of cancer) was investigated. Both response variables are binary, therefore logistic regression was performed, and corresponding $p$ values, are reported. Age was categorised by years into four groups: $<19,19-40,41-65$ and $>65$. We excluded the $<19$ age category from the regression analyses since it comprised a very small sample of patients and so including it would mask the effects of the other categories. Summarised descriptive statistics concerning ITU admission and mortality were generated in Table 1 (see supplementary material S1 for a fuller dataset).

\section{Results}

\section{General characteristics}

During the study period, 321 patients were hospitalised with COVID-19; age range was 14-104; median age was 73 years (interquartile range (IQR) $56-82$ ). The age groups in this cohort were $<19$ years $(0.6 \%) ; 19-40$ years $(7.2 \%) ; 41-65$ years $(30.5 \%)$ and $>65$ years $(61.7 \%)$; the eldest category representing the most frequently admitted with COVID-19. There were 132 (41\%) women and $189(59 \%)$ men; $246(77 \%)$ patients were of Caucasian origin whereas 75 (23\%) were of black, Asian and minority ethnic (BAME) groups.

Hospitalised COVID-19 patients' comorbidity history was of interest; 259 (80.6\%) had a comorbidity. Hypertension (123; $38.3 \%)$, heart disease $(86 ; 26.7 \%)$, diabetes $(64 ; 19.9 \%)$ and history of cancer $(47 ; 14.6 \%)$ were the most commonly reported comorbidities in these patients.

\section{Presentation}

The most commonly reported symptoms were shortness of breath in 202 patients (62.9\%), fever in 190 (59.1\%) and cough in 180 (56\%). Gastrointestinal symptoms were reported in 38 patients (11.8\%); of these, 15 (4.6\%) described abdominal pain, 13 (4\%) had diarrhoea and $15(4.6 \%)$ with nausea and vomiting. Mean body mass index was $24.2 \mathrm{~kg} / \mathrm{m}^{2}$. On admission, arterial oxygen level $\left(\mathrm{pO}_{2}\right)$ was measured on $131(40.8 \%)$ patients with a mean of $9.46 \mathrm{kPa}$ (normal $\mathrm{pO}_{2}$ range $11-14.4 \mathrm{kPa}$ ). Forty-four (13.7\%) patients had $\mathrm{pO}_{2}<8 \mathrm{kPa}$ signifying type 1 respiratory failure as a presenting feature.

Most of the patients (313; 97\%) had a chest X-ray on admission. Left-sided infiltrates were reported in $10.2 \%$, right-sided infiltrates in $10.2 \%$, bilateral infiltrates in $37.3 \%$ and $0.9 \%$ had pleural effusion. Only 21 patients had computed tomography (CT) of the chest during admission and, of these, 12 patients were reported to have multiple or limited areas of ground-glass shadowing in the lungs.

\section{ICU admission}

Decision to admit to ICU was made on expert advice from the critical care team in view of the patient's clinical status and the overall prognosis. The median length of ICU admission was 9.9 days (IQR 3-13). A total of $44(13.7 \%)$ received ICU care; 15 $(34.1 \%)$ were aged $>65,23(52.3 \%)$ were aged between 41 to 65 years, $5(11.4 \%)$ were aged between 19 and 40 years and w. $3 \%$ ) was aged $<19$ years. The patient aged $<19$ years had cancer and was undergoing chemotherapy. Being a very small sample, the $<19$ years category was excluded from all logistic regression analyses to avoid masking the effects of the other age categories. Thirtythree $(75 \%)$ patients admitted to ICU were males (male to female 
Table 1. Clinical characteristics and median admission blood tests of patients admitted with COVID-19 to University Hospital, Coventry, between 24 January 2020 and 13 April 2020

\begin{tabular}{|c|c|c|c|}
\hline & All inpatients, $n=321$ & ICU admission, $n=44$ & Non-survivors at discharge, $n=104$ \\
\hline Median age, years (IQR) & $73(56.0-82.0)$ & $59(49.0-68.0)$ & $80(72.0-88.0)$ \\
\hline Age $>65$, years $(\%)$ & $198(61.7)$ & $\begin{array}{l}15(34.1) \\
a_{p}=0.026\end{array}$ & $\begin{array}{l}88(84.6) \\
{ }^{b} p=0.011\end{array}$ \\
\hline Male, $n(\%)$ & $189(58.9)$ & $\begin{array}{l}33(75.0) \\
a_{p}=0.027\end{array}$ & $62(60.0)$ \\
\hline BAME, n (\%) & $75(23.3)$ & $\begin{array}{l}18(40.9) \\
a_{p}=0.008\end{array}$ & $19(18.2)$ \\
\hline Any comorbidity, n (\%) & $259(80.6)$ & $33(75.0)$ & $88(84.6)$ \\
\hline Heart disease, $n(\%)$ & $86(26.7)$ & $\begin{array}{l}4(9.0) \\
a_{p}=0.009\end{array}$ & $32(30.7)$ \\
\hline NLR (IQR) & $6.1(3.6-10.3)$ & $6.9(4.9-10.8)$ & $\begin{array}{l}7.2(4.6-13.0) \\
{ }^{b} p=0.028\end{array}$ \\
\hline CRP, mg/L (IQR) & $79(32.5-162.0)$ & $\begin{array}{l}149(64.0-215.0) \\
a_{p}<0.001\end{array}$ & $\begin{array}{l}129(51.0-222.0) \\
{ }^{b} p<0.001 \\
{ }^{c} p<0.001\end{array}$ \\
\hline Albumin, g/L (IQR) & $38(34.0-40.0)$ & $38(34.7-40.2)$ & $\begin{array}{l}37(32.0-39.0) \\
b_{p}=0.002 \\
c_{p}=0.005\end{array}$ \\
\hline
\end{tabular}

${ }^{a}=$ significant value for association with hospital care; ${ }^{b}=$ significant value for relationship with mortality; ${ }^{c}=$ significant value for association with mortality, adjusted for age; $B A M E$ = black, Asian and minority ethnic; $C R P=C$-reactive protein; $I C U$ = intensive care unit; $I Q R=$ interquartile range; $N L R=$ neutrophil:lymphocyte ratio.

ratio was 3:1) and 18 (40.9\%) were BAME. CRP levels were worse in patients admitted to ICU with median CRP level of $149 \mathrm{mg} / \mathrm{L}$ (IQR 64-215) in comparison with patients who were treated in the wards with median CRP level of $69.5 \mathrm{mg} / \mathrm{L}$ (IQR 29-142.5). Among those who were admitted to ICU, 22 (50\%) did not survive. More than half of ICU deaths, 13/22 (59\%) were BAME (five Asian, three black and five minority ethnic); the rest (nine) were Caucasian. Six deceased patients had no comorbidities with their age distribution being $50,57,67,71,80$ and 80 years.

There was a significant association between ICU attendance and BAME patients $(p=0.008)$ with odds ratio $(O R)$ of 2.5 and its $95 \%$ confidence interval (CI) being 1.3-4.9. Therefore, the odds for BAME patients' ICU admission was 2.5 times more than Caucasian patients, despite the fact that BAME patients had a median age of 56 years compared to 76.5 years for Caucasian patients admitted to ICU. The odds for males $(p=0.027$; OR 2.3; $95 \%$ CI 1.1-4.9) ICU admission was 2.3 times more than females. Age $>65$ years old ( $p=0.026$; OR 0.28 ; $95 \%$ CI $0.09-0.93$ ), heart disease ( $p=0.009$; OR 0.2; 95\% CI 0.1-0.6) and elevated CRP $(p<0.001$; OR 1.004; 95\% CI 1.002-1.008) were also significantly associated with admission to ICU. The $>65$ age category showed around four times higher odds than the 19 to 40 years category of being admitted to ICU, possibly because of their critical clinical conditions; similar to patients with heart disease whose ICU odds were five times less than the other patients. Also, each $1 \mathrm{mg} / \mathrm{L}$ increase in CRP increased the ICU odds by $0.4 \%$.

\section{Mortality}

Of the 321 patients, 104 died giving an overall mortality of $32.4 \%$ among the hospitalised patients. The overall median length of hospital stay was 8 days. The median age for non-survivors was 80 years (IQR 72-88) and for survivors was 66 years (IQR 52-79). Among the patients who did not survive, 88 (84.6\%) were aged $>65$ years, $12(11.5 \%)$ were aged $41-65$ years, three $(2.9 \%)$ were aged $19-40$ years and one (1\%) was aged $<19$. The patient aged $<19$ was admitted to ICU had cancer and was undergoing chemotherapy. Age $>65$ years old was associated with mortality $(p=0.011 ;$ OR $5 ; 95 \%$ CI 1.6-21.9). Thus, this age category was five times more likely to die in the hospital than the $19-40$ years category. Among non-survivors, the male to female ratio was 3:2 and the Caucasian to BAME ratio was 4.5:1.

Neutrophils $(p=0.047)$, neutrophil lymphocyte ratio (NLR; $p=0.028), \operatorname{CRP}(p<0.001)$ and albumin $(p=0.002)$ showed $a$ statistically significant association with mortality. When the model was adjusted for age, only CRP ( $p<0.001$; OR 1.006; $95 \%$ CI $1.004-1.008$ ) and albumin ( $p=0.005$; OR $0.94 ; 95 \%$ CI $0.90-0.98)$ were significantly associated with mortality and NLR was found to increase with age. When CRP was analysed categorically and the model adjusted for age, the odds for death for patients with a CRP $>160 \mathrm{mg} / \mathrm{L}$ was four times more than patients with CRP $<30 \mathrm{mg} / \mathrm{L}$ (OR 4.1; 95\% CI 2.0-8.8). Non-survivors had median CRP levels of $129 \mathrm{mg} / \mathrm{L}$ (IQR 51-222) compared to survivors with median CRP of 61.5 (IQR 25.7-125). Similarly, for albumin, the odds of dying for patients with albumin $>40 \mathrm{~g} / \mathrm{L}$ was $55 \%$ less than patients with albumin levels $<38 \mathrm{~g} / \mathrm{L}$ (OR $0.45 ; 95 \%$ CI 0.21-0.93). The non-survivors had median albumin of $37 \mathrm{~g} / \mathrm{L}$ (IQR 32.0-39.0) compared to survivors with median albumin of $38 \mathrm{~g} / \mathrm{L}$ (IQR 34.2-41.0). Stepwise backwards elimination was also performed, and similar results were obtained; inpatients $>65$ years $(p<0.001)$, high CRP $(p<0.001)$ and lower albumin $(p=0.05)$ were significantly associated with mortality. 
There was no significant relationship between BAME and death $(p=0.097)$ even when adjusted for age $(p=0.542)$; neither was there a significant relationship between mortality and being male $(p=0.880)$, even when adjusted for age $(p=0.828)$.

\section{Discussion}

COVID-19 currently remains a clinical challenge, with no approved curative antiviral treatment. Moreover, there is lack of prognostic factors of disease severity. NLR was shown as an independent risk factor for mortality in hospitalised COVID-19 patients in multivariate logistic models. ${ }^{14-16}$ This study found a statistically significant association between high NLR, raised CRP and low serum albumin at admission and mortality. However, when analysis was further adjusted for age, only albumin and CRP were found to be associated with mortality.

Currently, there are no standardised criteria for ICU admission. In this study, older age, high CRP, being BAME and male at hospital admission were significantly associated with ICU admission. Therefore, such parameters may be employed as risk factors for severe COVID-19, helping to identify patients requiring strict monitoring and possibly early therapeutic interventions.

Our study identified that COVID-19 main presenting symptoms were fever, cough and shortness of breath which is similar to the published literature. ${ }^{6,10}$ We also found that $11.8 \%$ of the patients reported one or more GI symptoms, including abdominal pain, diarrhoea and nausea/vomiting. This is comparable to study by Jin et al which identified that among 651 enrolled patients with COVID-19, 74 (11.4\%) presented with at least one GI symptom (nausea, vomiting or diarrhoea). ${ }^{17}$ Another study conducted by Lin et al reported GI symptoms in 58 out of $95(63.7 \%)$ patients which is more frequent in their cohort of patients. ${ }^{18}$

The Jin et al study identified that patients with GI symptoms were associated with increased rate of complications and critical disease $\left(22.9 \%\right.$ ) compared with COVID-19 with no GI symptoms. ${ }^{17}$ In contrast, our study showed a lower percentage with only $13.6 \%$ of patients who required ICU admission had GI symptoms. This variability in GI symptomatology of COVID-19 and association of severe critical disease, could be due to the different populations of the two studies. However, it is prudent to recognise GI symptoms with or without respiratory symptoms to ensure appropriate infection control measures within hospitals.

In this study, ICU admission rate was $13.7 \%$ which is consistent with previous studies. ${ }^{19-21}$ Death among ICU admissions was at $50 \%$, similar to the ICU death rate reported by the latest Intensive Care National Audit \& Research Centre (ICNARC) report issued on 30 April $2020 .^{22}$

The overall mortality of the admitted patients was $32.4 \%$ which is in line with a recent study, not peer-reviewed at the time of submission, evaluating 16,749 hospitalised UK patients with COVID-19 using the International Severe Acute Respiratory and Emerging Infection Consortium World Health Organization clinical characterisation protocol. The study reported a mortality rate of $33 \%{ }^{23}$ A systematic review by Rodriguez-Morales et al reported $13.4 \%$ case fatality rates among the hospitalised patients. ${ }^{19}$ However, the mean age of this meta-analysis (51 years) was much lower than our study. The older population profile, together with the presence of multiple comorbidities, could have contributed to our reported mortality rate. This was further confirmed by the strong association $(p<0.05)$ between age and death in our study population.

Emerging data from the USA, shows that ethnic minorities were at higher risk of COVID-19 infection in comparison to Caucasians. ${ }^{24}$ In the UK, $18 \%$ of total infected patients were BAME. $^{2}$ In England, Caucasians represent $80 \%$ of the whole population while BAME groups are around $20 \% .{ }^{25}$ The findings from our study showed that $23 \%$ of hospitalised COVID-19 patients were of BAME origin. Additionally, out of the 44 patients that required ICU admission, $40.9 \%$ were of BAME origin. There was a significant association between BAME patients and ICU admission ( $p=0.008)$. The mortality percentage of BAME patients in ICU was $59 \%$ (13/22 patients).

There was no significant relationship between BAME or being a male and death even when adjusted for age in this study. However, it is important to note that this an observational cohort with a relatively small number of patients and not truly representative of ethnicity as a major risk factor for death from COVID-19. This is because we did not have access to all patients' socioeconomic and educational factors from our hospital informatics system. The recently published data from the UK Office for National Statistics shows that the risk of death involving the COVID-19 among some ethnic groups to be significantly higher than that among Caucasian ethnicity. According to the study, when data were adjusted for age in the analysis, black men were 4.2 times more likely to die from COVID-19 and black women were 4.3 times more likely to die from COVID-19 than Caucasian men and women, respectively. People of Bangladeshi, Pakistani, Indian and mixed ethnicities also had a statistically significant raised risk of death involving COVID-19 compared with those of Caucasian ethnicity. ${ }^{26}$

COVID-19 has high mortality as demonstrated in our study. Globally the search for drugs and vaccines that could save lives is actively being looked into. A recent promising press release by the Randomised Evaluation of COVID-19 Therapy (RECOVERY) trial demonstrated that the use of the low-cost dexamethasone reduced death by up to one-third in ventilated patients and by one-fifth in other patients receiving oxygen only and that there was no benefit among those patients who did not require respiratory support. Although dexamethasone is not curative for COVID-19, this news will help inform clinical decision making in future patients with the disease. ${ }^{27}$

There were several limitations in our study. Firstly, selection bias could have occurred due to its retrospective observational nature. Secondly, no validated instruments were used for reporting symptoms. The data was collected from the hospital information system and it would have been influenced by subject variability with risk of certain symptoms being under-reported. This study focused only on patients who required hospital admission and thus the results cannot be generalised to the population.

\section{Conclusion}

Increasing age, CRP and low albumin were associated with poor outcomes (even after adjusting for age). NLR was also a potential marker of poor outcomes but its significance was lost after correcting for age. The above parameters could be implemented in clinical practice to identify high-risk patients of severe COVID-19 for close monitoring and management. Further validation from large cohorts is required. 


\section{Key points}

> COVID-19 inpatient hospital mortality in this study was high at $32.4 \%$.

$>$ GI symptoms occurred in $11.8 \%$ and do not seem to be associated with worse outcome.

$>$ Age $>65$ years, higher CRP and lower albumin on admission were associated with mortality.

> BAME, being male and high CRP are associated with ICU admission.

$>$ Admission NLR was elevated in non-survivors but when adjusted for age, logistic regression showed that elevated NLR is not significantly associated with mortality.

\section{Supplementary material}

Additional supplementary material may be found in the online version of this article at www.rcpjournals.org/clinmedicine: S1 - Full clinical characteristics of patients admitted with COVID-19.

\section{References}

1 World Health Organisation. Statement on the second meeting of the International Health Regulations (2005) Emergency Committee regarding the outbreak of novel coronavirus (2019-nCoV). Geneva: WHO, 2020. www.who.int/news-room/detail/30-01-2020-statement-on-the-second-meeting-of-the-international-health-regulations-(2005)-emergency-committee-regarding-the-outbreak-of-novel-coronavirus-(2019-ncov) [Accessed 07 May 2020].

2 NHS England. COVID-19 daily deaths. NHS. www.england.nhs.uk/ statistics/statistical-work-areas/covid-19-daily-deaths [Accessed 07 May 2020].

3 Liu Y, Gayle AA, Wilder-Smith A, Rocklöv J. The reproductive number of COVID-19 is higher compared to SARS coronavirus. J Travel Med 2020;27:taaa021.

4 Nishiura H, Linton NM, Akhmetzhanov AR. Serial interval of novel coronavirus (COVID-19) infections. Int J Infect Dis 2020;93:284-6.

5 Wu Y, Guo C, Tang L et al. Prolonged presence of COVID-19 viral RNA in faecal samples. Lancet Gastroenterol Hepatol 2020;5:434-5.

6 Guan W, Ni Z, Hu Y, Liang W, Ou C, He J, et al. Clinical characteristics of 2019 novel coronavirus infection in China. N Engl ] Med. 2020;382:1708-1720.

7 Lechien JR, Cabaraux P, Chiesa-Estomba CM et al. Objective olfactory evaluation of self-reported loss of smell in a case series of 86 COVID-19 patients. Head Neck 2020;42:1583-90.

8 Redd WD, Zhou JC, Hathorn KE et al. Prevalence and characteristics of gastrointestinal symptoms in patients with COVID-19 infection in the United States: A multicenter cohort study. Gastroenterology 2020. doi: 10.1053/j.gastro.2020.04.045 [Epub ahead of print].

9 Nobel YR, Phipps M, Zucker ] et al. Gastrointestinal symptoms and COVID-19: case-control study from the United States. Gastroenterology 2020. doi: 10.1053/j.gastro.2020.04.017 [Epub ahead of print].

10 Huang C, Wang Y, Li X et al. Clinical features of patients infected with 2019 novel coronavirus in Wuhan, China. Lancet 2020;395: 497-506.

11 Cheung KS, Hung IF, Chan PP et al. Gastrointestinal manifestations of COVID-19 infection and virus load in fecal samples from the Hong Kong cohort and systematic review and meta-analysis. Gastroenterology 2020;159:81-95.

12 Tian Y, Rong L, Nian W, He Y. Review article: gastrointestinal features in COVID-19 and the possibility of faecal transmission. Aliment Pharm Ther 2020;51:843-51.
13 Li Q, Ding X, Xia G et al. Eosinopenia and elevated C-reactive protein facilitate triage of covid-19 patients in fever clinic: a retrospective case-control study. EClinicalMedicine 2020;23:100375.

14 Yang A, Liu J, Tao W, Li H. The diagnostic and predictive role of NLR, d-NLR and PLR in COVID-19 patients. Int Immunopharmacol 2020;84:106504

15 Liu Y, Du X, Chen J et al. Neutrophil-to-lymphocyte ratio as an independent risk factor for mortality in hospitalized patients with COVID-19. J Infect 2020;81:e6-12.

16 Du RH, Liang LR, Yang CQ et al. Predictors of mortality for patients with COVID-19 pneumonia caused by COVID-19: A prospective cohort study. Eur Respir ] 2020;55:2000524.

17 Jin X, Lian JS, Hu JH et al. Epidemiological, clinical and virological characteristics of 74 cases of coronavirus-infected disease 2019 (COVID-19) with gastrointestinal symptoms. Gut 2020;69:1002-9.

18 Lin L, Jiang X, Zhang Z et al. Gastrointestinal symptoms of 95 cases with COVID-19 infection. Gut 2020;69:997-1001.

19 Rodriguez-Morales AJ, Cardona-Ospina JA, Gutiérrez-Ocampo E et al. Clinical, laboratory and imaging features of COVID-19: A systematic review and meta-analysis. Travel Med Infect Di 2020;34:101623.

20 Liang W, Guan W, Chen R et al. Cancer patients in COVID-19 infection: a nationwide analysis in China. Lancet Oncol 2020;31: 335-7.

21 Wang D, Hu B, Hu C et al. Clinical characteristics of 138 hospitalized patients with 2019 novel coronavirus-infected pneumonia in Wuhan, China. JAMA 2020;323:1061-9.

22 Intensive Care National Audit and Research Centre. Report on 2249 patients critically ill with COVID-19. ICNARC, 2020. www. icnarc.org/About/Latest-News/2020/04/04/Report-On-2249Patients-Critically-IIl-With-Covid-19 [Accessed 07 May 2020].

23 Docherty AB, Harrison EM, Green CA et al. Features of 16,749 hospitalised UK patients with COVID-19 using the ISARIC WHO clinical characterisation protocol. medRxiv www.medrxiv.org/content/10.11 01/2020.04.23.20076042v1.full.pdf [Accessed 07 May 2020].

24 Garg S, Kim L, Whitaker M et al. Hospitalization rates and characteristics of patients hospitalized with laboratory-confirmed coronavirus disease 2019- COVID-NET, 14 states, March 1-30, 2020. Weekly 2020;69:458-64. www.cdc.gov/mmwr/volumes/69/wr/ mm6915e3.htm [Accessed 07 May 2020].

25 Office for National Statistics. Population of England and Wales. England and Wales 2011 Census. ONS, 2019. www.ethnicityfacts-figures.service.gov.uk/uk-population-by-ethnicity/nationaland-regional-populations/population-of-england-and-wales/latest [Accessed 22 June 2020].

26 Office for National Statistics. Coronavirus (COVID-19) related deaths by ethnic group, England and Wales: 2 March 2020 to 10 April 2020. ONS, 2020. www.ons.gov.uk/peoplepopulationandcommunity/ birthsdeathsandmarriages/deaths/articles/coronavirusrelateddea thsbyethnicgroupenglandandwales/2march2020to10april2020 [Accessed 07 May 2020].

27 Chief investigators of the Randomised Evaluation of COVid-19 thERapY (RECOVERY) trial. Low-cost dexamethasone reduces death by up to one third in hospitalised patients with severe respiratory complications of COVID-19. RECOVERY, 2020. www.recoverytrial. net/news/low-cost-dexamethasone-reduces-death-by-up-to-onethird-in-hospitalised-patients-with-severe-respiratory-complicationsof-covid-19 [Accessed 22 June 2020].

Address for correspondence: Prof Ramesh P Arasaradnam, University Hospital, University Hospitals Coventry and Warwickshire NHS Trust, Clifford Bridge Road, Coventry CV2 2DX, UK.

Email: r.arasaradnam@warwick.ac.uk 


\section{Royal College \\ Q. of Physicians}

\section{Prolonged disorders of consciousness following sudden onset brain injury National clinical guidelines}

New guidelines from the Royal College of Physicians, endorsed or supported by a further

15 health bodies, offer updated guidance on the diagnosis, assessment, care and management of patients with prolonged disorders of consciousness.

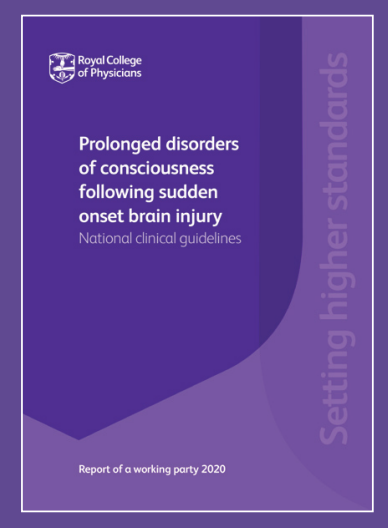

Download the updated guidelines www.rcplondon.ac.uk/pdoc 